\title{
Impact of CYP3A5(*)3 and CYP2C8-HapC on Paclitaxel/Carboplatin-Induced Myelosuppression in Patients with Ovarian Cancer
}

\author{
Henrik Green, Muhammad Suleman Khan, Ingrid Jakobsen-Falk, \\ Elisabeth Avall-Lundqvist and Curt Peterson
}

\section{Linköping University Post Print}

N.B.: When citing this work, cite the original article.

This is the authors' version of the following article:

Henrik Green, Muhammad Suleman Khan, Ingrid Jakobsen-Falk, Elisabeth Avall-Lundqvist and Curt Peterson, Impact of CYP3A5(*)3 and CYP2C8-HapC on Paclitaxel/CarboplatinInduced Myelosuppression in Patients with Ovarian Cancer, 2011, Journal of Pharmaceutical Sciences, (100), 10, 4205-4209.

which has been published in final form at:

http://dx.doi.org/10.1002/jps.22680

Copyright: Wiley-Blackwell http://eu.wiley.com/WileyCDA/Brand/id-35.html

Postprint available at: Linköping University Electronic Press http://urn.kb.se/resolve?urn=urn:nbn:se:liu:diva-71784 


\section{The Impact of CYP3A5*3 and CYP2C8-HapC on}

\section{Paclitaxel/Carboplatin-Induced Myelosuppression in Patients with}

\section{Ovarian Cancer}

Henrik Gréen ${ }^{1,2}$, Muhammad Suleman Khan ${ }^{1}$, Ingrid Jakobsen-Falk ${ }^{1}$, Elisabeth

Åvall-Lundqvist ${ }^{3}$, Curt Peterson ${ }^{1}$

${ }^{1}$ Clinical Pharmacology, Division of Drug Research, Faculty of Health Sciences, Linköping University, SE-581 85 Linköping, Sweden

${ }^{2}$ Science for Life Laboratory, School of Biotechnology, Division of Gene Technology, Royal Institute of Technology, SE-171 65 Solna, Sweden

${ }^{3}$ Department of Gynecologic Oncology, Radiumhemmet, Karolinska University Hospital, Solna, SE-171 76 Stockholm, Sweden

\section{Conflicts of interest}

The authors declare no conflicts of interest.

Running title: CYP3A5*3, CYP2C8-HapC and Paclitaxel

\section{Corresponding author:}

Henrik Gréen, PhD

Clinical Pharmacology

Department of Medical and Health Sciences

Faculty of Health Sciences

Linköping University

SE-581 85 Linköping

Sweden

E-mail: henrik.green@liu.se

Phone: +46101031544

Fax: +4613104195

Funding: This work was supported by grants from the Swedish Cancer Society, the Swedish Research Council, the European Commission (CHEMORES LSHC-CT2007-037665), and the Östergötland County Council. 
Abstract: The influence of genetic variants on paclitaxel-induced toxicity is of considerable interest for reducing adverse drug reactions. Recently the genetic variants CYP2C8*3, CYP2C8-HapC, and CYP3A5*3 were associated with paclitaxel-induced neurotoxicity. We therefore investigated the impact of CYP2C8HapC and CYP3A5*3 on paclitaxel/carboplatin-induced myelosuppression and neurotoxicity. Thirty-three patients from a prospective pharmacokinetics study were genotyped using pyrosequencing. Patients with variant alleles of CYP2C8HapC were found to have significantly lower nadir values of both leukocytes and neutrophils $(p<0.05)$ than wild type patients. CYP3A5*3/*1 patients were shown to have borderline significantly lower nadir values of leukocytes $(p=0.07)$ than *3/*3 patients. Combining the two genotypes resulted in a significant correlation with both leukopenia and neutropenia $(p=0.01)$. No effect of these genetic variants on the neurotoxicity could be shown in this rather small study, but their importance for paclitaxel-induced toxicity could be confirmed.

\section{INTRODUCTION}

Paclitaxel in combination with carboplatin is the standard chemotherapy regimen in ovarian cancer, but it is associated with significant myelosuppression and neurotoxicity. The predictability of the adverse effects therefore has considerable clinical significance. Paclitaxel is metabolized by CYP2C8 to $6-\alpha-$ hydroxypaclitaxel and to p-3'-hydroxypaclitaxel by CYP3A4/3A $5^{1-5}$. Polymorphisms in these genes may influence the clinical effects and pharmacokinetics of paclitaxel and several single nucleotide polymorphisms (SNPs) have been investigated. It has been demonstrated that CYP2C8*3 is associated with reduced clearance of total ${ }^{6}$ and unbound ${ }^{7}$ paclitaxel and that the 
same allele has been associated with sensory neurotoxicity. ${ }^{6,8}$ However, other groups have not been able to demonstrate the same association. ${ }^{9-10}$ Leskelä et al. recently found an association between paclitaxel-induced neurotoxicity and the genetic variants CYP2C8*3, CYP2C8-HapC, and CYP3A5*3. ${ }^{8}$ To validate these results, we investigated the impact of CYP2C8-HapC and CYP3A5*3 on paclitaxel/carboplatin-induced myelosuppression and neurotoxicity in a previously

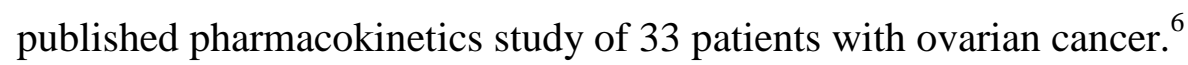

\section{MATERIAL AND METHODS}

The patients included in this study were treated with $175 \mathrm{mg} / \mathrm{m}^{2}$ (or $135 \mathrm{mg} / \mathrm{m}^{2}, \mathrm{n}=$ 3) of paclitaxel plus carboplatin (AUC $=5$ or 6 ) every three weeks for at least six cycles, except for one patient who only received four cycles due to severe neurotoxicity. ${ }^{6}$ Myelosuppression was evaluated as the nadir leukocyte and neutrophil counts during the whole treatment period and according to CTC (Common Terminology Criteria) scale version 2. In addition to CTC scale assessment, 23 patients underwent a more detailed evaluation of sensory neuropathy using the method of Cassidy et al., ${ }^{11}$ which generated N-scores of 0 to 17 on a neurotoxicity severity scale. The genotypes were investigated by Pyrosequencing $^{\circledR}$ as previously described, ${ }^{6}$ but with new primers. The forward primer bio-CTTAGATTTGCATTTTGAGACTAT, the reverse primer CAGCAGAAGAAAGAATTAGTGAG, and the sequencing primer GAAAGAATTAGTGAGCTTTAAC were used for CYP2C8-HapC (rs1113129, G>C). To determine the CYP2C8-HapC genotype, the nucleotides were added in the following dispensation order in the Pyrosequencer ${ }^{\circledR}$ : GACAGCGTA. The bioATGTACCACCCAGCTTAA forward primer and ACACACAGCAAGAGTCTCA 
reverse primer were used to amplify the region around CYP3A5*3 (rs776746, $A>G)$. The sequencing primer GGTCCAAACAGGGAA was annealed to the template and the dispensation order CGAGATACTCG was used to determine the CYP3A5*3 genotype of the patients. The association of the CYP3A5*3 and CYP2C8-HapC genotypes with toxicity was tested using Fisher's exact test when evaluating the toxicity according to the CTC scale. The Mann-Whitney U test was used to compare the $\mathrm{N}$-score and nadir values of leukocytes and neutrophils between genotypes.

\section{RESULTS}

Five of the 33 patient were heterozygous for CYP3A5*3 and 28 were *3/*3. As regards CYP2C8-HapC, two patients were homozygous for the genetic variant, 11 were heterozygous and the rest were wild-type. For CYP2C8-HapC, the toxicity found in the wild-type patients was compared with that found in the other patients. A significant association was found between myelosuppression according to the CTC scale and both CYP3A5*3 and CYP2C8-HapC genotypes. CYP3A5*3 homozygously variant patients showed a significantly lower risk of developing leukopenia ( $p=0.01$, Fisher's exact test) than heterozygous $* 3 / * 1$ patients. Five out of 28 patients in the $* 3 / * 3$ group developed severe (CTC grade $3-4$ ) leukopenia, compared to four out of five patients in the *3/*1 group (Table 1 ). A similar trend was found for $* 3 / * 3$ genotypes regarding neutropenia, but this was not statistically significant. CYP2C8-HapC variants *1/C + C/C were associated with a significant ( $p=0.01$, Fisher's exact test) increased risk of developing neutropenia compared to wild-type patients $(* 1 / * 1)$. Ten out of 13 patients in the $* 1 / C+C / C$ group suffered from severe life-threatening neutropenia (CTC grade 3-4), compared to six out of 
20 patients in the $* 1 / * 1$ group (Table 1 ). A trend towards an increased risk of leukopenia was also associated with CYP2C8-HapC, although this was not statistically significant. We could not find an association between neuropathy according to the CTC scale and either CYP2C8-HapC or CYP3A5*3 in this population.

The nadir values of leukocytes and neutrophils during the whole treatment were compared between the different genotypes of CYP3A5*3 and CYP2C8-HapC (Figure 1). Patients with CYP2C8-HapC *1/*1 had significantly higher median leukocyte and neutrophil nadir counts than patients with $* 1 / C+C / C(p=0.03$ and $p=0.02$, respectively). A similar trend difference in median nadir values for leukocytes and neutrophils was also observed between the different genotypes of CYP3A5*3. Patients with the CYP3A5*3/*3 genotype tended to have borderline significantly higher median nadir leukocyte and neutrophil values than $* 3 / * 1$ patients ( $p=0.07$ and $p=0.14$, respectively). We also combined the genotypes so that patients with the CYP3A5*3/*3 and CYP2C $8 * 1 / * 1$ genotypes were compared with the rest of the patients. Using this strategy of combined genotypes, patients with the CYP3A5*3/*3 and CYP2C8*1/*1 genotypes showed significantly higher nadir values of leukocytes $(p=0.01)$ and neutrophils $(p=0.01)$ as compared to the rest of the patients. 


\section{DISCUSSION}

Our data are consistent with the data published by Leskelä et al. ${ }^{8}$ in the sense that both CYP3A5*3 and CYP2C8-HapC are associated with adverse reactions to paclitaxel therapy. Like Leskelä et al., we found less toxicity in patients with the CYP3A5*3/*3 genotype than in the wild-type patients. However, we found that carriers of the CYP2C8-HapC variant had a higher risk of neutropenia, in contrast to Leskelä et al. who found a lower risk of neuropathy in this group of patients. ${ }^{8}$ This may either be due to the different types of toxicity being studied or to dose changes made in response to the acquired toxicity, which could have prevented the development of the other form of toxicity in that particular patient. Paclitaxel is a neurotoxic drug in a dose-dependent manner and this adverse event is also the doselimiting toxicity. However, both paclitaxel and carboplatin cause myelosuppression, so the leukopenia and neutropenia seen in this study could have been caused by either of these drugs. We have previously found that CYP2C8*3 is associated with increased neuropathy ${ }^{6}$ in accordance with the findings of Leskelä et al. ${ }^{8}$ Both CYP2C8*3 ${ }^{6-7}$ and CYP2C8-HapC ${ }^{12}$ have previously been shown to be associated with a lower clearance of paclitaxel, which may explain the higher degree of toxicity in these patients. Leskelä et al. also put forward a hypothesis that metabolites may affect the tissues and be associated with the adverse drug reactions, without themselves being cytotoxic. ${ }^{13}$ This could explain why CYP3A5*3, which is associated with a splice variant resulting in a low CYP3A5 activity, ${ }^{14}$ is also associated with low toxicity. Our toxicity data are, in fact, consistent with this hypothesis, as we found that the genotypes associated with low CYP2C8 and high CYP3A5 activity are associated with myelosuppression. We have also previously shown that a change in metabolic activity may be associated 
with a shift in the metabolic patterns in patients treated with paclitaxel. ${ }^{6}$ Leskelä et al. found an association between CYP2C8*3 and neuropathy, ${ }^{8}$ in accordance with our previous data. ${ }^{6}$ However, we could not confirm an association between neuropathy and either CYP2C8-HapC or CYP3A5*3 in our small series. A final genetic model was presented by Leskelä et al. combining the CYP3A5*3, CYP2C8-HapC, and CYP2C8*3 genotypes. When using this model in our series (CYP2C8*3 data from our previous study ${ }^{6}$ ), we could not find any significant association with toxicity (data not shown); however, combining CYP3A5*3 and CYP2C8-HapC resulted in a significant association with myelosuppression.

Previous studies have suggested that there is an association between genotype and paclitaxel-induced toxicities. As mentioned above, both Gréen et al. ${ }^{6}$ and Leskelä et $a l .{ }^{8}$ found associations between the genotypes of the metabolizing enzymes and toxicity. However, studies by Henningsson et al. ${ }^{10}$ and Marsh et al. ${ }^{9}$ did not find an association between toxicity and the ABCB1, CYP2C8, or CYP3A5 genotypes. These discrepancies may be due to the use of a wide range of infusion times and dosages, the use of a combination of docetaxel and paclitaxel, lack of adjustment for the dose-dependent nature of paclitaxel-induced neuropathy, and non-inclusion of the functional CYP2C8-HapC genotype.

In conclusion, these results show that genotyping may be a feasible approach for the individualization of paclitaxel chemotherapy and that the CYP2C8-HapC and CYP3A5*3 alleles are SNPs of interest. However, a large part of the clinical response to paclitaxel cannot currently be explained by these genotypes alone. New candidate genes and larger studies are necessary to elucidate the true impact of patient genotype on the individual response to paclitaxel therapy. 
Table 1. Comparison between CYP3A5*3 and CYP2C8-HapC genotypes and toxicity associated with paclitaxel treatment in ovarian cancer

\begin{tabular}{|c|c|c|c|c|c|c|c|c|}
\hline \multirow{2}{*}{ Toxicity } & \multirow[t]{2}{*}{ CYP3A5 } & \multicolumn{2}{|c|}{ CTC Grading } & \multirow[t]{2}{*}{$p$ value } & \multirow{2}{*}{ CYP2C8-HapC } & \multicolumn{2}{|c|}{ CTC Grading } & \multirow[t]{2}{*}{$p$ value } \\
\hline & & $0-2, n$ & $3-4, n$ & & & $0-2, n$ & $3-4, n$ & \\
\hline \multirow[t]{2}{*}{ Leukopenia } & $\star 3 / * 3$ & 23 & 5 & & $* 1 /{ }^{*} 1$ & 17 & 3 & \\
\hline & $* 3 / * 1$ & 1 & 4 & 0.01 & ${ }^{*} 1 / C+C / C$ & 7 & 6 & 0.11 \\
\hline \multirow[t]{3}{*}{ Neutropenia } & $* 3 / * 3$ & 16 & 12 & & $* 1 / * 1$ & 14 & 6 & \\
\hline & $* 3 / * 1$ & 1 & 4 & 0.17 & ${ }^{*} 1 / C+C / C$ & 3 & 10 & 0.01 \\
\hline & & $0, \mathrm{n}$ & $1-2, n$ & & & $0, n$ & $1-2, n$ & \\
\hline \multirow[t]{2}{*}{ Neurosensory } & $* 3 / * 3$ & 15 & 12 & & $* 1 / * 1$ & 11 & 9 & \\
\hline & $* 3 / * 1$ & 5 & 0 & 0.13 & ${ }^{*} 1 / C+C / C$ & 9 & 3 & 0.27 \\
\hline
\end{tabular}

CTC $=$ Common Terminology Criteria for adverse events, $* 3 / * 3=$ homozygously variant patients, ${ }^{*} 3 / * 1=$ heterozygous patients, ${ }^{* 1} /{ }^{*} 1=$ wild type, ${ }^{*} 1 / \mathrm{C}+\mathrm{C} / \mathrm{C}=$ heterozygous + homozygously variant patients, $\mathrm{n}=$ No. of patients. 
Figure
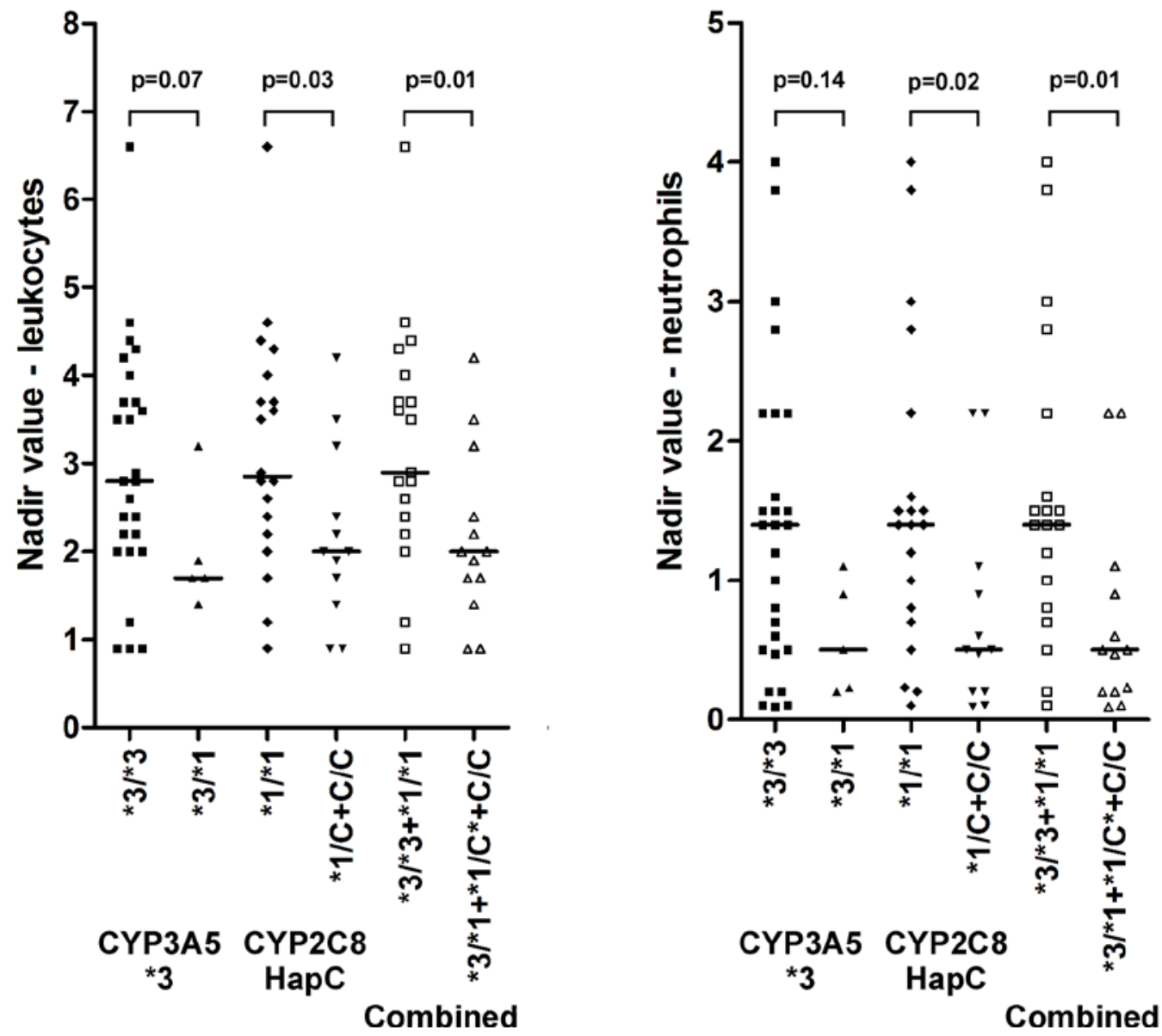

Figure 1. Comparison of the nadir values of leukocytes and neutrophils during paclitaxel treatment in ovarian cancer patients depending on the CYP3A5*3 and CYP2C8-HapC genotypes. Patients carrying the CYP $3 A 5^{*} 3 /{ }^{*} 3$ genotype were compared with the patients carrying the ${ }^{*} 3 /{ }^{*} 1$ genotype and patients carrying one or two variants of the CYP2C8-HapC were compared with the rest of the patients. The CYP3A5*3 and CYP2C8-HapC genotypes were also combined so that patients carrying both CYP $3 A 5^{\star} 3 / * 3$ and CYP2C $8 * 1 / * 1$ were compared with the rest of the patients, i.e. those carrying at least one of the CYP $3 A 5^{\star} 1$ or CYP2C8-HapC alleles. The two patients with that were homozygously variant for CYP2C8HapC had leukocyte nadir values of 3.5 and 4.2, and neutrophil nadir value of 2.2 and 2.2 cells $/ \mathrm{mm}^{3}$. 


\section{References}

1. Vaclavikova R, Soucek P, Svobodova L, Anzenbacher P, Simek P, Guengerich FP, Gut I 2004. Different in vitro metabolism of paclitaxel and docetaxel in humans, rats, pigs, and minipigs. Drug Metab Dispos 32:666-674.

2. Rahman A, Korzekwa KR, Grogan J, Gonzalez FJ, Harris JW 1994. Selective biotransformation of taxol to 6 alpha-hydroxytaxol by human cytochrome P450 2C8. Cancer Res 54:5543-5546.

3. Harris JW, Rahman A, Kim BR, Guengerich FP, Collins JM 1994. Metabolism of taxol by human hepatic microsomes and liver slices: participation of cytochrome P450 3A4 and an unknown P450 enzyme. Cancer Res 54:40264035.

4. Cresteil T, Monsarrat B, Alvinerie P, Treluyer JM, Vieira I, Wright M 1994. Taxol metabolism by human liver microsomes: identification of cytochrome P450 isozymes involved in its biotransformation. Cancer Res 54:386-392.

5. Walle T, Walle UK, Kumar GN, Bhalla KN 1995. Taxol metabolism and disposition in cancer patients. Drug Metab Dispos 23:506-512.

6. Green H, Soderkvist P, Rosenberg P, Mirghani RA, Rymark P, Lundqvist EA, Peterson C 2009. Pharmacogenetic studies of Paclitaxel in the treatment of ovarian cancer. Basic Clin Pharmacol Toxicol 104:130-137.

7. Bergmann TK, Brasch-Andersen C, Green H, Mirza M, Pedersen RS, Nielsen F, Skougaard K, Wihl J, Keldsen N, Damkier P, Friberg LE, Peterson C, Vach W, Karlsson MO, Brosen K 2011. Impact of CYP2C8*3 on paclitaxel clearance: a population pharmacokinetic and pharmacogenomic study in 93 patients with ovarian cancer. Pharmacogenomics J 11:113-120.

8. Leskela S, Jara C, Leandro-Garcia LJ, Martinez A, Garcia-Donas J, Hernando S, Hurtado A, Vicario JC, Montero-Conde C, Landa I, Lopez-Jimenez E, Cascon A, Milne RL, Robledo M, Rodriguez-Antona C 2011. Polymorphisms in cytochromes P450 2C8 and 3A5 are associated with paclitaxel neurotoxicity. Pharmacogenomics J 11:121-129.

9. Marsh S, Paul J, King CR, Gifford G, McLeod HL, Brown R 2007. Pharmacogenetic assessment of toxicity and outcome after platinum plus taxane chemotherapy in ovarian cancer: the Scottish Randomised Trial in Ovarian Cancer. J Clin Oncol 25:4528-4535.

10. Henningsson A, Marsh S, Loos WJ, Karlsson MO, Garsa A, Mross K, Mielke S, Vigano L, Locatelli A, Verweij J, Sparreboom A, McLeod HL 2005. Association of CYP2C8, CYP3A4, CYP3A5, and ABCB1 polymorphisms with the pharmacokinetics of paclitaxel. Clin Cancer Res 11:8097-8104.

11. Cassidy J, Paul J, Soukop M, Habeshaw T, Reed NS, Parkin D, Kaye SB 1998. Clinical trials of nimodipine as a potential neuroprotector in ovarian cancer patients treated with cisplatin. Cancer Chemother Pharmacol 41:161-166.

12. Rodriguez-Antona C, Niemi M, Backman JT, Kajosaari LI, Neuvonen PJ, Robledo M, Ingelman-Sundberg M 2008. Characterization of novel CYP2C8 haplotypes and their contribution to paclitaxel and repaglinide metabolism. Pharmacogenomics J 8:268-277.

13. Sparreboom A, Huizing MT, Boesen JJ, Nooijen WJ, van Tellingen O, Beijnen JH 1995. Isolation, purification, and biological activity of mono- and 
dihydroxylated paclitaxel metabolites from human feces. Cancer Chemother Pharmacol 36:299-304.

14. Kuehl P, Zhang J, Lin Y, Lamba J, Assem M, Schuetz J, Watkins PB, Daly A, Wrighton SA, Hall SD, Maurel P, Relling M, Brimer C, Yasuda K,

Venkataramanan R, Strom S, Thummel K, Boguski MS, Schuetz E 2001.

Sequence diversity in CYP3A promoters and characterization of the genetic basis of polymorphic CYP3A5 expression. Nat Genet 27:383-391. 